\title{
DISC TOOL PROFILING FOR AIR COMPRESSOR SCREWS WITH COMPLEX CHARACTERISTIC CURVES
}

\begin{abstract}
One of the best practical methods to machine the air compressor screw pairs is milling or grinding by disc tools. The mathematics involved in disc tool profiling is quite complex and requires representing the machined surface by mathematical equations without any singular points and undercutting. From a technical perspective, this article proposes the disc tool profiling integrated solution for machining complex profile screws from their reverse engineering data, which contains crucial issues such as machining deviation evaluation due to undercutting, appropriate tool position setting. The disc tool surface designed by the proposed method is highly accurate, leading the RMS error of the machined surface is less than $0.0201 \mathrm{~mm}$. A difference of the angle, formed by the disc tool axis and the workpiece axis, less than $0.16^{\circ}$, is acceptable in air compressor technology, which leads to a machined surface RMS deviation of less than $0.0186 \mathrm{~mm}$.
\end{abstract}

\section{INTRODUCTION}

Screw air compressors have been widely used since 1975 due to their advantages such as compactness, high performance, and stability. The main reasons for these advantages are creating new rotor profiles, which have reduced internal leakage, and the appearance of precise thread milling machines at the same time.

One of the best practical methods to machine the air compressor screw pairs is milling or grinding by disc tools. The traditional methods for disc tool profiling involve specifying the contact conditions and creating the characteristic curve. The analytical methods [1-2] for disk tool profiling, based on traditional envelope theory, have been widely used for a long time. Besides, numerical-analytical methods [3-7] were also proposed recently.

The mathematics involved in the above methods is quite complex, so it cannot be solved directly but requires a dedicated algorithm. Besides, these methods require a representation of the machined surface by mathematical equations and without any singular points and undercutting. Actually, disk tool profiling is often supported by expensive dedicated software based on advanced methods, which are still secret and only suitable for large companies.

\footnotetext{
${ }^{1}$ Faculty of Mechanical Engineering, Ha Noi University of Industry, Viet Nam

${ }^{2}$ School of Mechanical Engineering, Ha Noi University of Science and Technology, Viet Nam

*E-mail: long.hoang@ hust.edu.vn https://doi.org/10.36897/jme/140054
} 
One nowadays advanced trend is the CAD approach, such as using the "Projection" command on CATIA to project the disc-tool axis onto the machined surface to get characteristic curve [8], machining simulation and identification of contact lines by Boolean operation [9-10], and the method of "relative generating trajectories" [11-13] but these works were written not in detail and almost ignored the problem of undercutting as well as the impact of disc tool position on the accuracy of the air compressor screw pair.

Existing problems that need to be considered are:

- The analytical method is complex, powerless in the case of undercutting or singular point.

- Analytical method can only solve accurately in some exceptional cases, and it must be solved generally by approximate numerical methods using programming.

- The analytical method requires a surface representation through a system of equations, which would be inconvenient with reverse engineering.

- The method using the perpendicular projection of the disc tool axis onto the machined surface has not been shown in detail, especially when undercutting or singular points appear.

- Boolean method is slow when performing, cannot be used in reverse engineering.

The paper aims to solve the shortcomings above by proposing the disc tool profiling integrated solution for machining complex profile screws from reverse engineering data, which contains crucial issues such as machining deviation evaluation due to undercutting, appropriate tool position setting.

\section{PROPOSED SOLUTIONS FOR DISC TOOL PROFILING AND MACHINED SURFACE DEVIATION EVALUATION}

\subsection{FUNDAMENTAL THEORY IN BRIEF}

Litvin [1] denotes $\Sigma_{1}$ and $\Sigma_{2}$ for the generating and generated surfaces, respectively. The applied coordinate systems $\mathrm{S}_{1}, \mathrm{~S}_{2}$, are connected to $\Sigma_{1}, \Sigma_{2}$, respectively. $\Sigma_{1}$ is represented as vector function $r_{l}(u, \theta)$ where $u, \theta$ are surface general mathematical parameters (such as angle and displacement of a point on the surface $\Sigma_{1}$ in the system $\mathrm{S}_{1}$ ). Using the coordinate transformation from $S_{1}$ to $S_{2}$, the family of surfaces $\Sigma_{1}$ is represented in $S_{2}$ as vector function $r_{2}(u, \theta, \tau)$, where $\tau$ is the motion parameter.

The envelope $\Sigma_{2}$ is tangent to all surfaces of the family of surface $r_{2}(u, \theta, \tau) . \Sigma_{1}$ and $\Sigma_{2}$ must have a common tangent plane. This requirement deduces the meshing equation as [1].

$$
\left(\partial r_{2} / \partial u \times \partial r_{2} / \partial \theta\right) \times\left(\partial r_{2} / \partial \tau\right)=0
$$

The meshing condition of the screw pair and their cutting tool are exceptional cases of crossed helical gears. Based on the theory of enveloping surfaces, the meshing condition of a crossed helical gear pair (see Fig. 1) is written by Stosic [5] as below:

$$
\begin{gathered}
{\left[C-x_{1}+\left(p_{1}-p_{2}\right) \cdot \cot \Sigma\right]\left(x_{1} \cdot \partial x_{1} / \partial t+y_{1} \cdot \partial y_{1} / \partial t\right)+} \\
p_{1}\left[\left(\mathrm{v} \cdot p_{1} \theta \partial y_{1} / \partial t+\left(p_{2}-C \cdot \cot \Sigma\right) \partial x_{1} / \partial t\right)\right]=0
\end{gathered}
$$




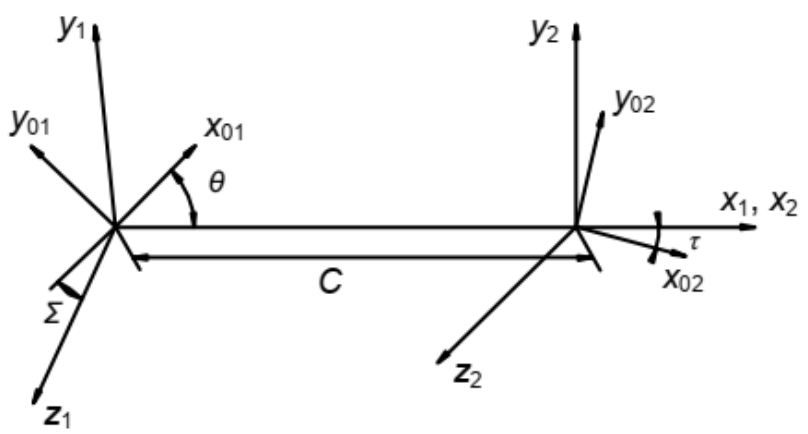

Fig. 1. The coordinate system of helical gears with non-parallel and non-intersecting axes, where $x_{01}, y_{01}$, and $x_{02}, y_{02}$ are coordinates of the points on the gear end cross-section

These coordinate systems are fixed to gear 1 and gear $2 . \Sigma$ is the rotation angle around the $x$-axis of the system $x_{1} y_{1} z_{1}$ compared with the system $x_{2} y_{2} z_{2} ; p_{1}$ and $p_{2}$ are unit leads of gear 1 and gear 2, respectively. $\theta$ and $\tau$ are rotation angles of gear 1 and gear 2 around their axis. $C$ is the distance between these gear axes.

Where $x_{01}, y_{01}$, and $x_{02}, y_{02}$ are coordinates of the points on the gear end cross-section. These coordinate systems are fixed to gear 1 and gear $2 . \Sigma$ is the rotation angle around the $x$-axis of the system $x_{1} y_{1} z_{1}$ compared with the system $x_{2} y_{2} z_{2} ; p_{1}$ and $p_{2}$ are unit leads of gear 1 and gear 2, respectively. $\theta$ and $\tau$ are rotation angles of gear 1 and gear 2 around their axis. $C$ is the distance between these gear axes.

The problem of specifying air compressor screw profiles is solved by placing $\Sigma=0$ into Eq. (2), the resulting meshing equation, in this case, become as [5]:

$$
d x_{o 1} / d y_{o 1} \cdot\left(k y_{o 1}-C / i \cdot \sin \theta\right)+k x_{o 1}+C / i \cdot \cos \theta=0
$$

where $i=p 2 / p 1, k=1-1 / i$.

Placing $p_{2}=0$ into Eq. (2), disc tools (see Fig. 2a) can be determined as [5]:

$$
\left(C-x_{1}+p_{1} \cdot \cot \Sigma\right)\left(x_{1} \cdot \partial x_{1} / \partial t+y l \cdot \partial y_{1} / \partial t\right)+p_{1} \cdot\left(p_{1} \cdot \theta \cdot \partial y_{1} / \partial t-C \cdot \cot \Sigma \cdot \partial x_{1} / \partial t\right)=0
$$

\subsection{PROPOSED SOLUTIONS FOR DISC TOOL PROFILING AND MACHINED SURFACE DEVIATION EVALUATION}

Based on Eq. (1), the characteristic curve of both the generated surface and the disc-tool surface is determined as [8]:

$$
\left(\vec{A}, \vec{N}_{\Sigma}, \vec{r}_{1}\right)=0
$$

where: $\vec{A}$ is disc tool axis, $\vec{N}_{\Sigma}$ is a vector normal to the generating helical surface, $\vec{r}_{1}$ is a position vector of a point on the helical surface.

From Eq. (5), it can be proved that the characteristic curve is the perpendicular projection of the disc tool axis onto the generated surface. Using the projection command in CATIA, the characteristic curve and disc tool are created, as shown in Fig. $2 \mathrm{~b}$ below. 
The characteristic curve and disk tool creating process is shown in Fig. 3 below. In the process, the reference plane is created parallel to the heliacal surface axis. $\Sigma$ and $h$ are position parameters of the disc tool axis on the reference plane. If the characteristic curve is discontinuous, the parameters $\Sigma$ and $h$ are modified to meet the continuous property requirement.

a)

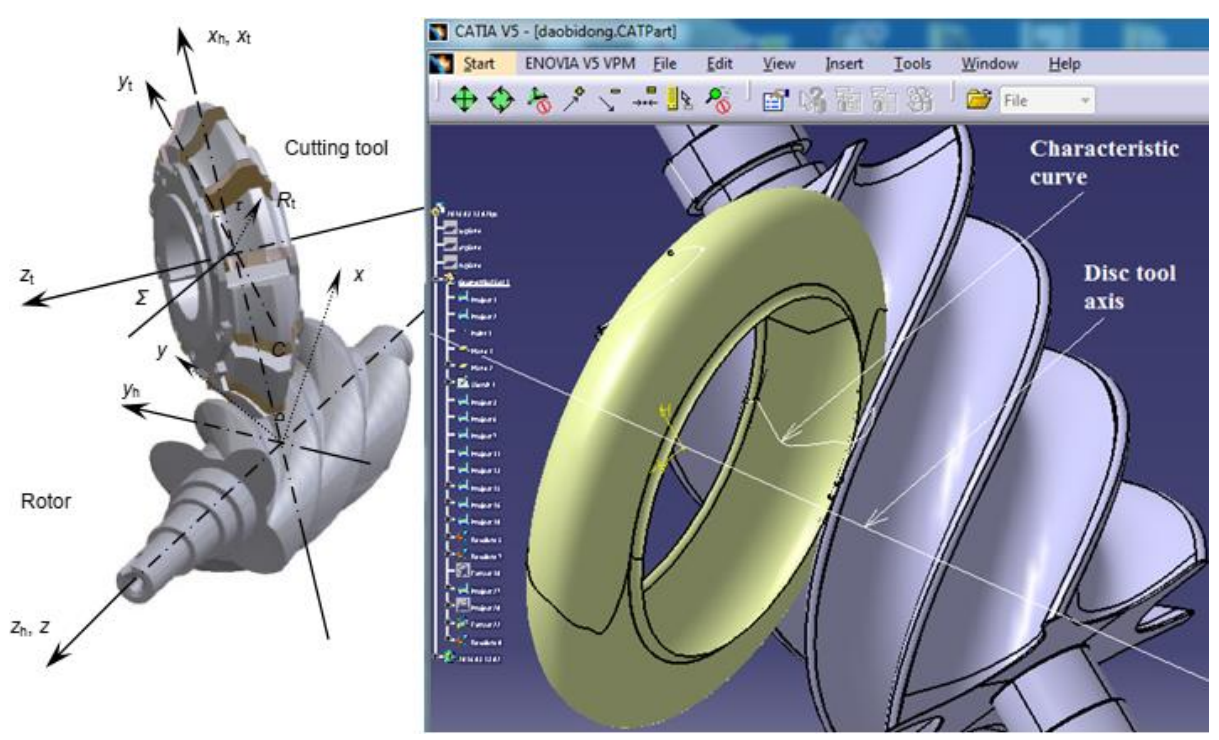

Fig. 2. a) Disc tool and Rotor coordinate systems [5], b) characteristic curve was created by projecting disc tool axis onto the helical surface

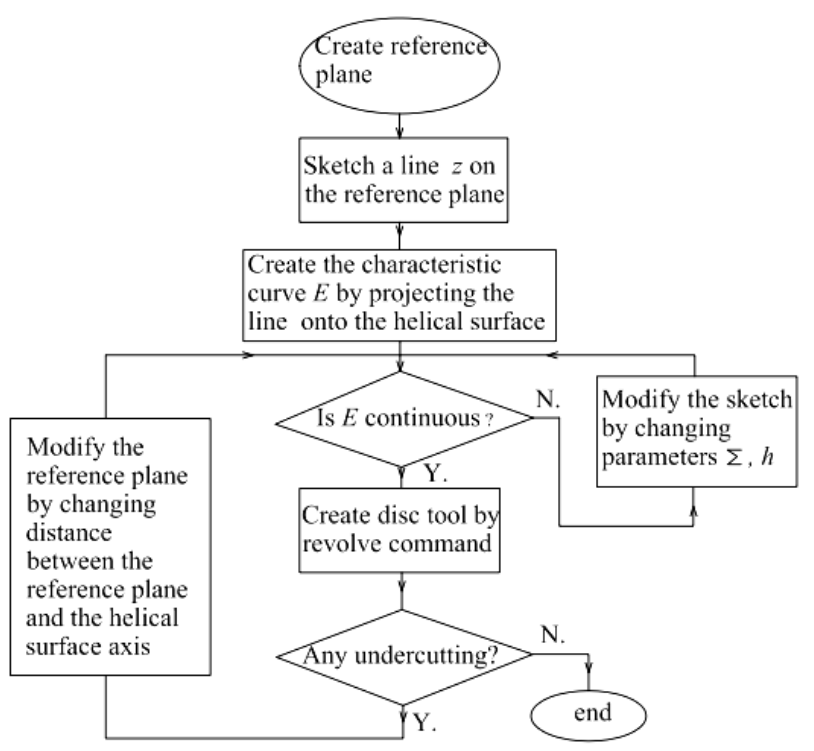

Fig. 3. The characteristic curve and disk tool creating process

Verifying the accuracy of the machined rotor surface and its disc tool was performed by simulative machining as follows. 
The simulative machining procedure has been written as an AutoLISP subroutine running in AutoCAD to generate the helical surface. The procedure is performed by a loop, including the "Subtract" command, to cut out step by step the "workpiece" when the disc tool is moved and rotate along the helical path (for more information, see our paper [14]).

Using Geomagic Control X, a 3D comparison between the 3D scanned helical surface and the helical surface generated by the disc tool is created to show the machined surface deviation (see Fig. 7).

\section{EXPERIMENTAL RESULT AND DISCUSSION}

The air compressor Airman PDS50 rotor pair ware scanned by using the Nikon MMDX100 3D digital handheld scanner then the IGS data file was exported into Solid Work as shown in Figs. 4 and 5. (The accuracy of such CAD models is about $15 \mu \mathrm{m}$, as shown in Figs. 4 and 5. To correct these models, see our previous works, such as [14], for more information).

Execute the process in Fig. 3 for creating a characteristic curve and disk tool of the main rotor (it is more difficult than the gate rotor), the distance between the disc tool axis and workpiece axis is $120 \mathrm{~mm}$, the angle $\Sigma$ formed by the two axes is $36.314^{\circ}$. In this position of the cuter, the characteristic curve is nearly continuous without a singular point. The result is shown in Fig. 6.

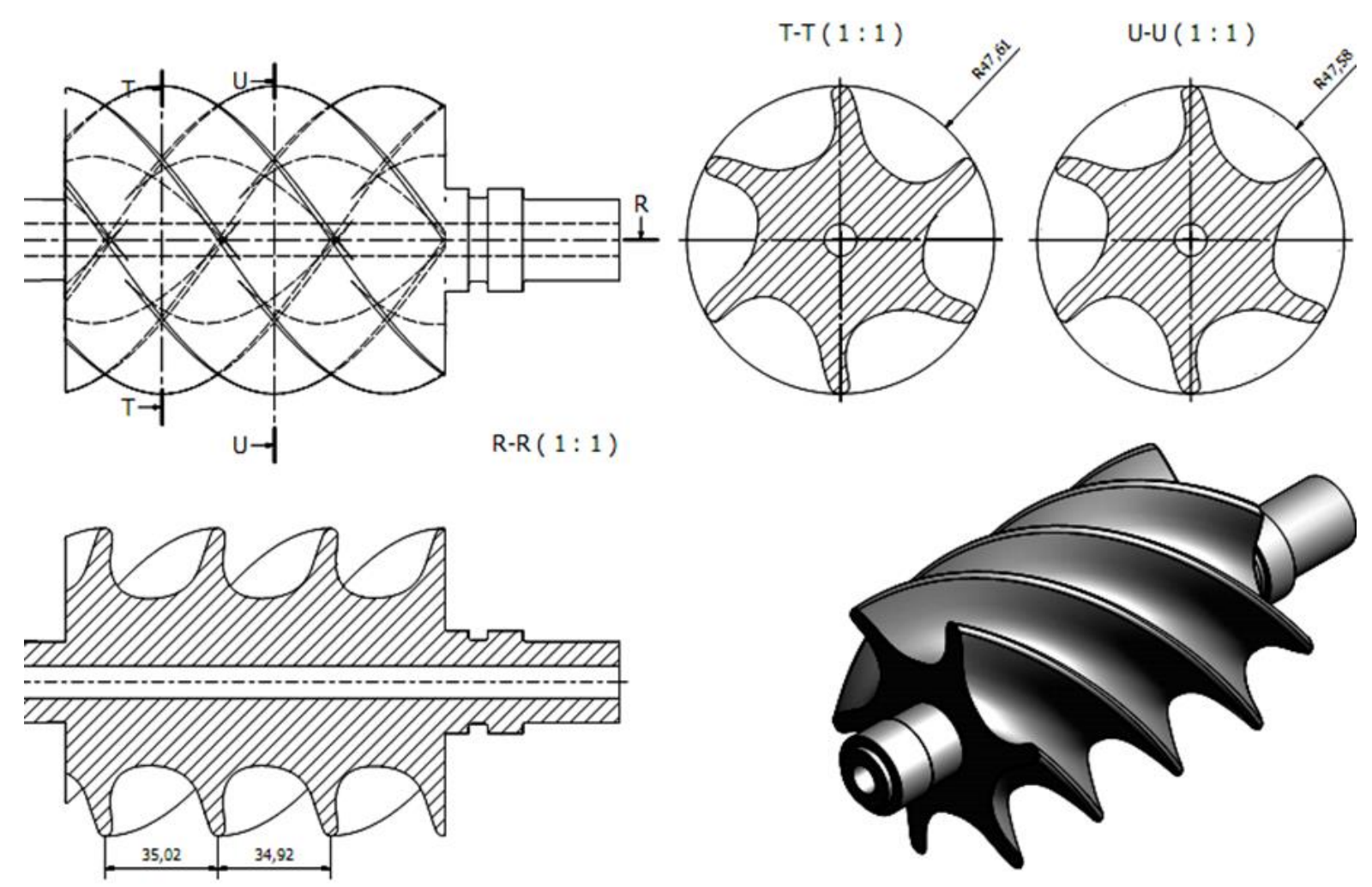

Fig. 4. 3D scanned model of the gate rotor 


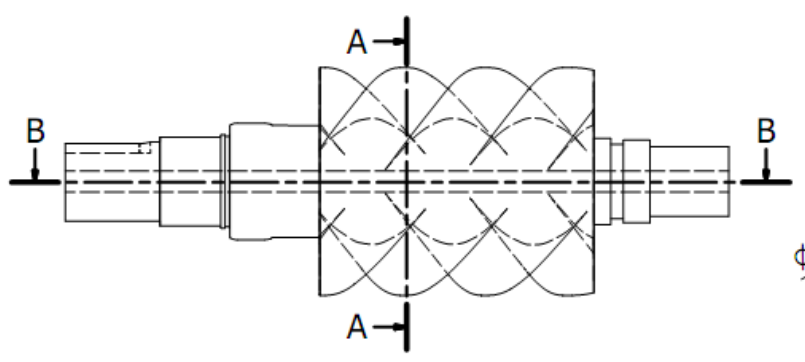

A-A ( $1: 2)$

B-B $(1: 2)$
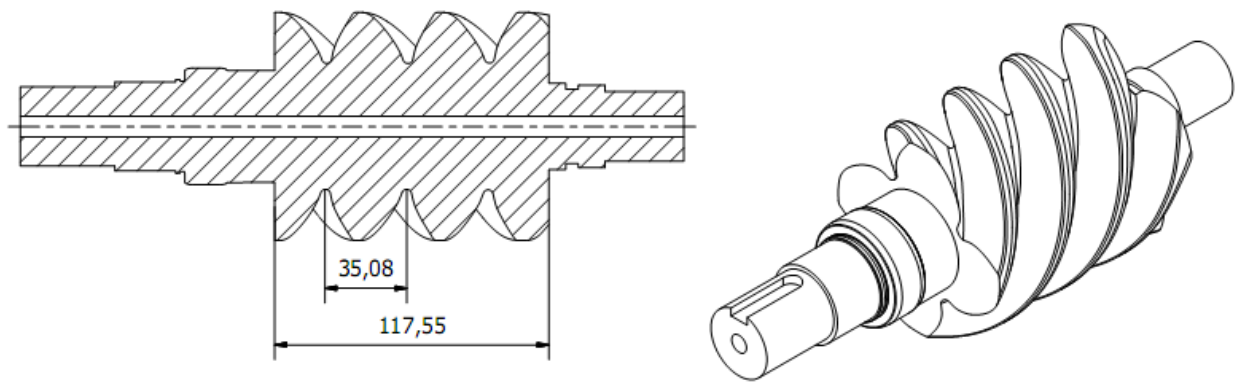

Fig. 5. 3D scanned model of the main rotor

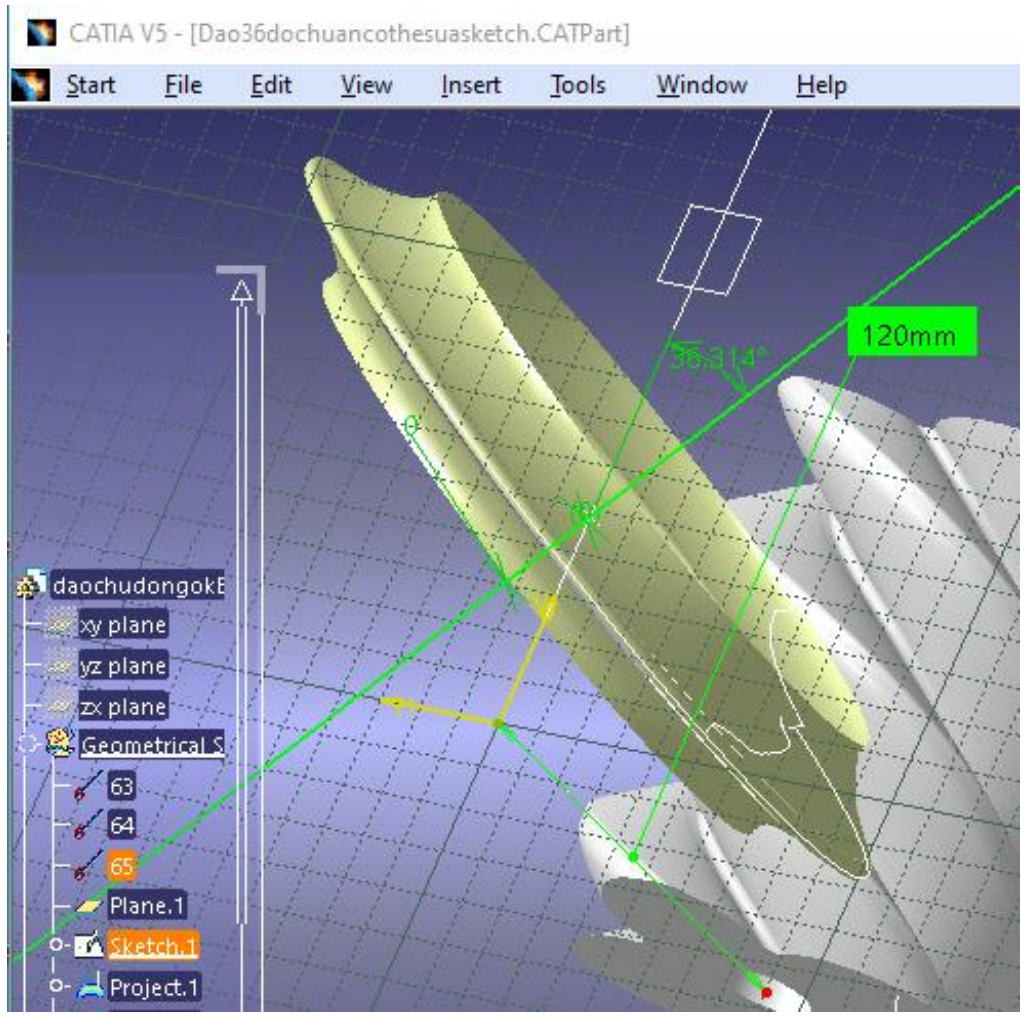

Fig. 6. Disc tool and the main rotor in the appropriate position

Using Geomagic Control X, a 3D comparison of the rotor surface created by revert engineering, which was imported as IGS reference data, and the helical surface generated by the disc tool, which was imported as STL measured data, is shown in Fig. 7. In the 3D comparison, the best fit alignment between the two models was used. 
The figure demonstrates that the disc tool surface created by the proposed method is highly accurate (the RMS error of the surface machined by the disc tool is $0.02 \mathrm{~mm}$.
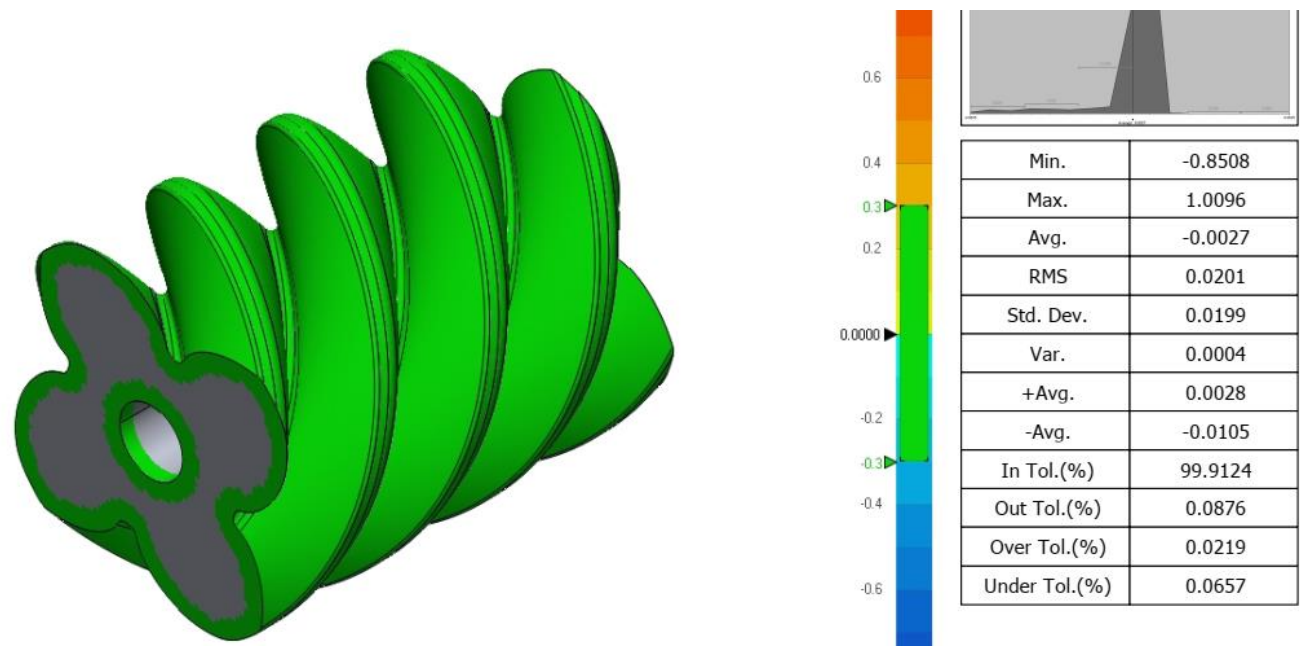

Fig. 7. The 3D accuracy of the helical surface machined by the disc tool (the used unit is $\mathrm{mm}$ )

Changing the angle $\Sigma$ from $36.314^{\circ}$ to $36.00^{\circ}$ and then $36.160^{\circ}$ leads to the disc tool surface difference, measured by the Geomagic Control X, as shown in Fig. 8 and Fig. 9, respectively. The disc tool surface difference logically represents the $3 \mathrm{D}$ deviation of the machined helical surface when the angle $\Sigma$ is changed due to cuter position setting error or low machine hardness.

Figure 8 shows that when the angle difference is $0.314^{\circ}$, the RMS error is $0.0339 \mathrm{~mm}$, which is not accepted in air compressor technology.

When the angle difference is $0.16^{\circ}$, the RMS error is $0.0186 \mathrm{~mm}$ (see Fig. 9), that is near to be accepted in air compressor technology. The analysis above can be used to select the machine that has enough accuracy and hardness.
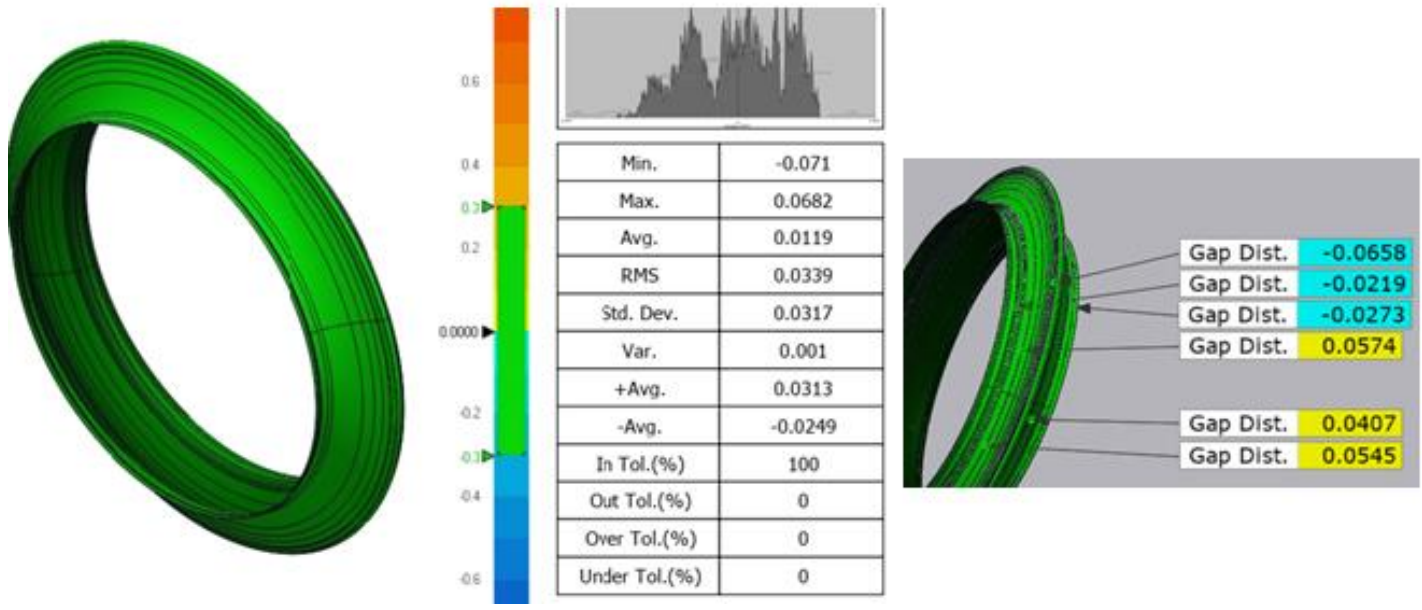

Fig. 8. 3D comparison of two disc-tools in the angles $\Sigma$ of $36.314^{\circ}$ and $36^{\circ}$ (the used unit is mm) 

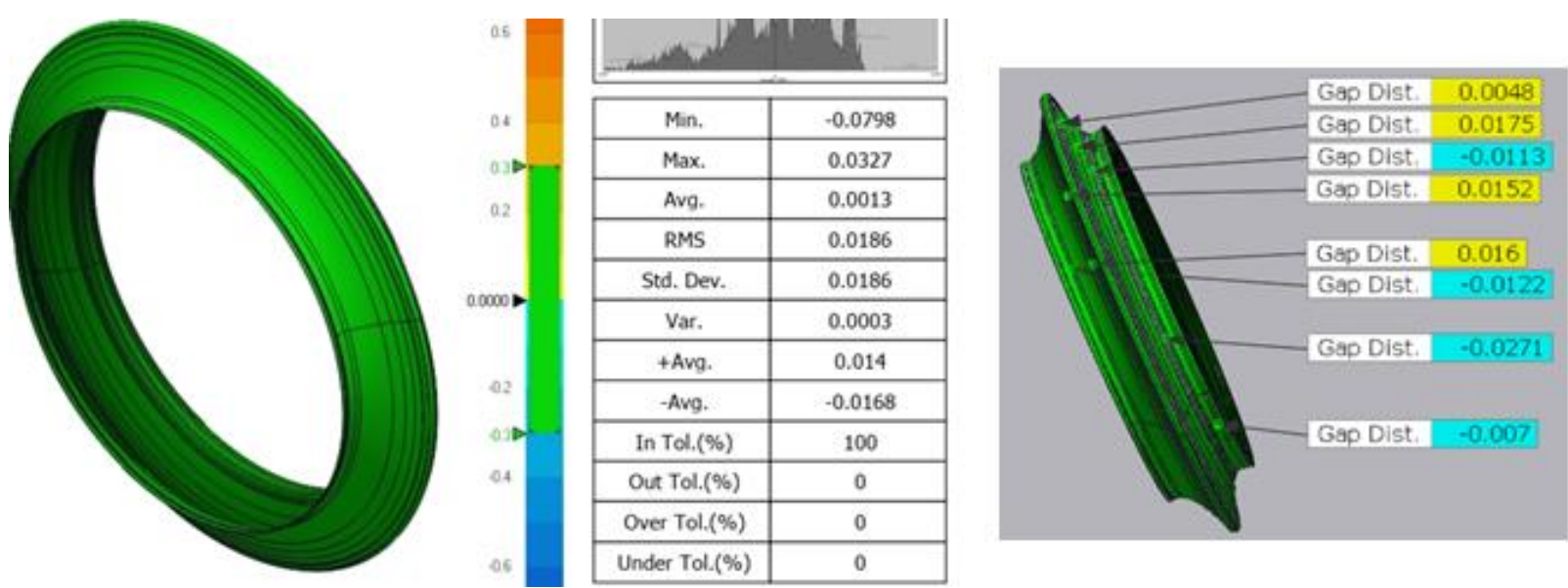

Fig. 9. 3D comparison of two disc-tools in the angles $\Sigma$ of $36.314^{\circ}$ and $36.16^{\circ}$ (the used unit is mm)

\section{CONCLUSION}

The research results of this work lead to the following conclusions:

1. The angle formed by the disc tool axis and the workpiece axis strongly influences the undercutting phenomenon.

2. The proposed process (in Fig. 3) is helpful to choose the appropriate value of this angle: for the main rotor of the air compressor AIRMAN PDF 50, the best angle is around the value of $36.314^{\circ}$.

3. The proposed methods for disc tool profiling and machined surface deviation evaluation are high accuracy, easy to implement without knowledge of complex envelope theory as traditional methods.

4. A surface deviation indirectly evaluation through tool deviation evaluation is faster and more comfortable than direct assessment.

5. A difference of the angle, formed by the disc tool axis and the workpiece axis, less than $0.16^{\circ}$, is acceptable in air compressor technology with a machined surface RMS deviation of less than $0.0186 \mathrm{~mm}$.

\section{REFERENCES}

[1] LITVIN F.L., 1997, Development of Gear Technology and Theory of Gearing, NASA Center for AeroSpace Information, 301, 621-0390, https://apps.dtic.mil/dtic/tr/fulltext/u2/a341717.pdf.

[2] VELIKO I., GENTCHO N., 1998, Profiling of Rotation Tools for Forming Helical Surfaces, Int. J. Mach. Tools Manuf., 38, 1125-1148.

[3] STOSIC N., 2006, A Geometric Approach to Calculating Tool Wear in Screw Rotor Machining, International Journal of Machine Tools \& Manufacture, 46, 1961-1965.

[4] STOSIC N., 2006, Evaluating Errors in Screw Rotor Machining by Tool to Rotor Transformation, Proc. IMechE Part B, J. Engineering Manufacture, 220, 1589-1596, DOI: 10.1243/09544054JEM552.

[5] STOSIC N., SMITH I.K., KOVACEVIC A., MUJIC E., 2011, Geometry of Screw Compressor Rotors and their Tools, Journal of Zhejiang University-SCIENCE A (Applied Physics \& Engineering), ISSN 1673-565X (Print), ISSN 1862-1775 (Online), 12/4, 310-326. 
[6] OANCEA N., POPA I., TEODOR V., OANCEA V., 2010, Tool Profiling for Generation of Discrete Helical Surfaces, Int. J. of Adv. Manuf. Technol., 50, 37-46.

[7] TEODOR V.G., POPA I., OANCEA N., 2010, The Profiling of end Mill and Planning Tools to Generate Helical Surfaces Known by Sampled Points, Int. J. Adv. Manuf. Technol., 51, 439-452.

[8] FRUMUSANU G., BERBINSCHI S., OANCEA N., 2011, Disc Tool Profiling - a Comparison Between the CAD Method and the Analytical Method, Proceedings in Manufacturing Systems, 6, 37-42.

[9] MOHAN L.V., SHUNMUGAM M.S., 2004, CAD Approach for Simulation of Generation Machining and Identification of Contact Lines, International Journal of Machine Tools \& Manufacture, 44, 717-723.

[10] MOHAN M.S., SHUNMUGAM L.V., 2007., Simulation of Whirling Process and Tool Profiling for Machining of Worms, Journal of Materials Processing Technology, 185, 191-197.

[11] BERBINSCHI S., TEODOR V.G., OANCEA N., 2012, 3D Graphical Method for Profiling Tools that Generate Helical Surfaces, Int. J. Adv. Manuf. Technol., 60, 505-512, DOI 10.1007/s00170-011-3637-3.

[12] BERBINSCHI S., TEODOR V.G., OANCEA N., 2012, A Study on Helical Surface Generated by the Primary Peripheral Surfaces of Ring tool, Int. J. V. Adv. Manuf. Technol., 61,15-24, DOI 10.1007/s00170-011-3687-6.

[13] BAROIU N., TEODOR V.G., SUSAC F., PĂUNOIU V., OANCEA N., 2019, The Planning Tool's Profiling for Roots Compressor's Rotors, Graphical Method in Catia, International Journal of Modern Manufacturing Technologies, XI/1, 7-13, ISSN 2067-3604.

[14] HOANG L., BANH T.L., NGUYEN T.L., 2020, A New Method for Designing and Machining Air Compressor Screw Pairs, International Journal of Modern Physics B, 34, 1-5, https://doi.org/10.1142/S0217979220401281. 